\title{
Accuracy assessment of the scalar network analyzer using sliding termination techniques
}

Knudsen, Bent; Engen, Glenn F.; Guldbrandsen, Birthe

Published in:

I E E E Transactions on Instrumentation and Measurement

Link to article, DOI:

10.1109/19.192331

Publication date:

1989

Document Version

Publisher's PDF, also known as Version of record

Link back to DTU Orbit

Citation (APA):

Knudsen, B., Engen, G. F., \& Guldbrandsen, B. (1989). Accuracy assessment of the scalar network analyzer using sliding termination techniques. I E E E Transactions on Instrumentation and Measurement, 38(2), 480483. https://doi.org/10.1109/19.192331

\section{General rights}

Copyright and moral rights for the publications made accessible in the public portal are retained by the authors and/or other copyright owners and it is a condition of accessing publications that users recognise and abide by the legal requirements associated with these rights.

- Users may download and print one copy of any publication from the public portal for the purpose of private study or research.

- You may not further distribute the material or use it for any profit-making activity or commercial gain

- You may freely distribute the URL identifying the publication in the public portal

If you believe that this document breaches copyright please contact us providing details, and we will remove access to the work immediately and investigate your claim. 


\title{
Accuracy Assessment of the Scalar Network Analyzer Using Sliding Termination Techniques
}

\author{
BENT KNUDSEN, MEMBER, IEEE, GLENN F. ENGEN, FELLOW, IEEE, AND BIRTHE GULDBRANDSEN
}

\begin{abstract}
In the absence of phase response the major, if not the primary, sources of error in the scalar network analyzer are the imperfect directivity, etc., associated with its internal and frequently "inaccessible" test set or measurement network. This paper obtains an explicit expression for this error in terms of the observed response to a sliding termination and sliding short.
\end{abstract}

\section{INTRODUCTION}

$\mathrm{T}$ HE APPLICATION of automation techniques to the field of microwave metrology is perhaps best exhibited by the "vector"' automated network analyzer (ANA) including the "six-port." At the same time, there is continuing interest in the "scalar" ANA. As implied by the name, and in contrast to the vector ANA, this device generally provides amplitude measurements only, and is based on a simpler detector and measurement network (or "test set"). It is also subject to certain sources of error (e.g., imperfect coupler directivity) due to its nonideal test set which, in principle at least, are eliminated in the vector ANA by the use of the phase detection capability which is included therein.

The evaluation of these error sources poses a challenge for the metrology laboratory in that (except for the "test port") the terminals of the measurement network (test set) are not ordinarily available. The problem is thus to ascertain what can be inferred about their magnitude in terms of the system response to certain terminations.

The role of the sliding termination in microwave metrology is a well-known and important one. An example is provided by the vector network analyzer where the phase response permits a "complete" evaluation of the test set parameters. Their effect on the observed response may then be eliminated by the software. The existing calibration techniques, by which these parameters are determined, usually include observations of the system response to a weakly reflecting sliding termination (or "load"). This permits one to make a projection of the system response to a totally nonreflecting termination. In

Manuscript received June 10, 1988.

B. Knudsen is with the Electromagnetics Institute, Technical University of Denmark, Lyngby 2800, Denmark.

G. F. Engen was with the Electromagnetics Institute, Technical University of Denmark, Lyngby, Denmark. He is now with Boulder Research Inc., Boulder, $\mathrm{CO}$.

G. Guldbrandsen is with the Institute of Circuit Theory and Telecommunication, Technical University of Denmark, Lyngby 2800 , Denmark. IEEE Log Number 8826207. addition, certain of these calibration methods also call for the use of sliding "shorts" as well.

In an earlier era, both the sliding load and the sliding short played a major role in the adjustment of the "tuned" reflectometer. This measurement technique provided for in situ adjustments of the hardware parameters, leading to an "ideal" test set; realization of this test set was recognized by the attainment of a constant ratio between the two detector readings in response to the motion of the sliding load and the sliding short.

In the absence of phase response, the basic theory for the scalar network analyzer is the same as that for the (tuned) reflectometer, but where the prescribed tuning criteria have not been completely realized. Thus certain of the test set parameters, referred to above, now become sources of error. Although the literature contains several investigations of this problem, these are less complete than the treatment to be given here.

It is the purpose of this paper to obtain limits for these errors in terms of the observed failure to satisfy the prescribed tuning conditions. The algebraic expression, which represents the error evaluation, is obtained first by a "conventional" analysis and then by the use of a computer program. This has made possible a more complete error expression than would have otherwise been obtained.

\section{BASIC THEORY}

As shown in Fig. 1, the basic form of the scalar network analyzer is a reflectometer whose two detectors respond to amplitude only. Denoting these by $P_{3}$ and $P_{4}$, one has the well-known result

$$
\frac{P_{3}}{P_{4}}=|a|^{2} \cdot \frac{\left|\Gamma_{U}+b\right|^{2}}{\left|c \Gamma_{U}+1\right|^{2}}
$$

where $\Gamma_{U}$ represents the "unknown" reflection coefficient at the test port, and $a, b$, and $c$ are complex parameters which describe the reflectometer or test set. By hypothesis, $|b|$ and $|c|$ are small and $\left|\Gamma_{U}\right| \leq 1$ so that, approximately:

$$
\frac{P_{3}}{P_{4}}=|a|^{2} \cdot\left|\left(\Gamma_{U}+b\right)\left(1-c \Gamma_{U}+c^{2} \Gamma_{U}^{2}+\cdots\right)\right|^{2}
$$




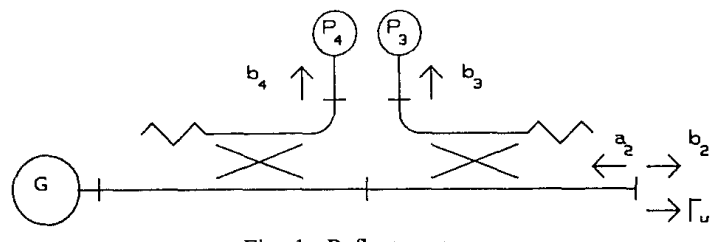

Fig. 1. Reflectometer.

which may be further expanded to yield

$$
\frac{P_{3}}{P_{4}}=|a|^{2} \cdot\left(\left|\Gamma_{U}\right|^{2}+b^{*} \Gamma_{U}+b \Gamma_{U}^{*}+|b|^{2}\right) \cdot K
$$

where

$$
\begin{aligned}
K= & {\left[1-c \Gamma_{U}-c * \Gamma_{U}^{*}+\left|c \Gamma_{U}\right|^{2}\right.} \\
& \left.+\left(c \Gamma_{U}\right)^{2}+\left(c * \Gamma_{U}^{*}\right)^{2}+\cdots\right]
\end{aligned}
$$

and the superscript $*$ represents the complex conjugate.

The reflection of the sliding termination will be denoted by $\Gamma_{L}$. In terms of the complex wave amplitudes, $b_{3}, b_{4}$ (the square of whose magnitudes are $P_{3}$ and $P_{4}$ ), the counterpart to (1) may be written

$$
\frac{b_{3}}{b_{4}}=a \cdot \frac{\Gamma_{L}+b}{c \Gamma_{L}+1}
$$

or, alternatively, as may be confirmed by a little algebra and letting $w=b_{3} / b_{4}$

$$
w=\frac{a\left(b-c^{*}\left|\Gamma_{L}\right|^{2}\right)}{1-|c|^{2}\left|\Gamma_{L}\right|^{2}}+\frac{a(1-b c) \Gamma_{L}}{1-|c|^{2}\left|\Gamma_{L}\right|^{2}} \cdot \frac{1+c^{*} \Gamma_{L}^{*}}{1+c \Gamma_{L}} .
$$

By hypothesis, the sliding termination provides a reflection coefficient of constant magnitude but variable phase. Thus the locus of $w$, in response to the sliding termination, may be written as the sum of two terms where the first is a constant while the second is of constant amplitude but variable argument. (Note that the second factor in the second term is of magnitude unity.) The locus of $w$ is thus a circle of radius $R$ and center $R_{C}$, which are given by

$$
\begin{aligned}
R & =\frac{|a||1-b c|\left|\Gamma_{L}\right|}{1-|c|^{2}\left|\Gamma_{L}\right|^{2}} \\
R_{C} & =\frac{a\left(b-c^{*}\left|\Gamma_{L}\right|^{2}\right)}{1-|c|^{2}\left|\Gamma_{L}\right|^{2}}
\end{aligned}
$$

For a weakly reflecting sliding load, $\left|\Gamma_{L}\right| \ll<1$. Adding the subscript $L,(6)$ and (7) may be written approximately as

$$
\begin{aligned}
R_{L} & \simeq|a|\left|\Gamma_{L}\right| \\
R_{C L} & \simeq a b
\end{aligned}
$$

since by hypothesis $\left|\Gamma_{L}\right|$ and $|b c|$ are small. Using the subscript $S$ to represent the sliding short, and assuming
$\left|\Gamma_{S}\right| \simeq 1$, one has

$$
R_{S} \simeq|a|
$$

and

$$
R_{C S} \simeq a\left(b-c^{*}\right)
$$

since $|c|^{2}$ and $|b c|$ are small with respect to unity.

Let

$$
d=b-c^{*}
$$

which is thus proportional to $R_{C S}$. After solving (12) for $c$ and substituting in (3), one has, to the first order in $b$ and $d$,

$$
\begin{aligned}
\frac{P_{3}}{P_{4}} \simeq & |a|^{2}\left[\left|\Gamma_{U}\right|^{2}+\left(b \Gamma_{U}^{*}+b^{*} \Gamma_{U}\right)\left(1-\left|\Gamma_{U}\right|^{2}\right)\right. \\
& \left.+\left(d \Gamma_{U}^{*}+d^{*} \Gamma_{U}\right)\left|\Gamma_{U}\right|^{2}+|b|^{2}+\cdots\right]
\end{aligned}
$$

where the second-order term $|b|^{2}$ has also been retained since this characterizes the error as $\Gamma_{U} \rightarrow 0$.

To continue, (9)-(12) may be combined to yield

$$
|b| \simeq \frac{\left|R_{C L}\right|}{R_{S}}
$$

and

$$
|d| \simeq \frac{\left|R_{C S}\right|}{R_{S}}
$$

which provide (approximate) values for $|b|$ and $|d|$ in terms of the observable parameters $\left|R_{C L}\right|,\left|R_{C S}\right|$, and $R_{S}$. In general the accompanying arguments are unknown; thus assuming a worst-case phase relationship in (13), one has

$$
\begin{aligned}
\frac{P_{3}}{P_{4}}= & \left|a \Gamma_{U}\right|^{2}\left[1 \pm 2\left|\frac{R_{C L}}{R_{S}}\right| \frac{1-\left|\Gamma_{U}\right|^{2}}{\left|\Gamma_{U}\right|} \pm 2\left|\frac{R_{C S}}{R_{S}}\right|\left|\Gamma_{U}\right|\right. \\
& \left.+\left|\frac{R_{C L}}{R_{S}}\right|^{2} \frac{1}{\left|\Gamma_{U}\right|^{2}}\right]
\end{aligned}
$$

which is the result of interest.

Assuming $|a|$ is known, that $\left|\Gamma_{U}\right| \gg\left|R_{C L} / R_{S}\right|$, and that $\left|\Gamma_{U}\right| \gg\left|R_{C S} / R_{S}\right|,(16)$ implicitly describes the error in a measurement of $\left|\Gamma_{U}\right|$ due to nonzero values of $R_{C L}$ and $R_{C S}$. In particular, by use of the \pm signs, one may obtain limits for $\left|\Gamma_{U}\right|$. On the other hand, as may be confirmed by (1), if $\left|\Gamma_{U}\right| \rightarrow 0$, one has $\left(P_{3} / P_{4}\right)=|a b|^{2}$ and the response no longer depends on $\Gamma_{U}$. In the interval where $\left|\Gamma_{U}\right|=\left|R_{C L} / R_{S}\right|$, both the first- and second-order terms in $\left|R_{C L} / R_{S}\right|$ are important, and an aid in interpreting (16) is obtained as follows. By further use of (5), and referring to Fig. 2, for small values of $\left|\Gamma_{U}\right|$ the response, $w$, will be given approximately by the vector sum of $R_{C}$ (or $R_{C L}$ ) and $a \Gamma_{U}$, but where the angle between them is unknown. By inspection

$$
|| R_{C}|-| w|| \leq\left|a \Gamma_{U}\right| \leq\left|R_{C}\right|+|w| .
$$




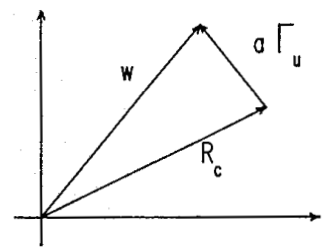

Fig. 2. Aid for interpreting equation (16)

In general there are two cases where $\left|\Gamma_{U}\right|$ is " well defined," $|w| \ll\left|R_{C}\right|$ and $|w| \gg\left|R_{C}\right|$. The first of these will only be obtained, however, if $\Gamma_{U}$ happens to very nearly equal the negative of $R_{C L} / a$ and is of only limited interest. The alternative where $|w / a|$, and thus $\left|\Gamma_{U}\right|$, is substantially (perhaps a factor of 10 or more) larger than $\left|R_{C L} / a\right|$ has been considered above. In this case, the term which contains the factor $\left|R_{C L} / R_{S}\right|^{2}$ can be neglected since the error is now dominated by the two terms in $\left|R_{C L} / R_{S}\right|$ and $\left|R_{C S} / R_{S}\right|$ in (16). In particular, if $\left|\Gamma_{U}\right|$ is still "small," say of the order of 0.1 , the major source of error will be due primarily to the first of these if $\left|R_{C L}\right|$ and $\left|R_{C S}\right|$ are nominally equal. For example, if $\left|R_{C L} / R_{S}\right|=0.01$, and $\left|\Gamma_{U}\right|=0.1$, one has a nominal 20 -percent error due to the first, and 0.2 percent from the second. As $\left|\Gamma_{U}\right|$ increases, the error from the first term diminishes, and vanishes in the limits as $\left|\Gamma_{U}\right| \rightarrow 1$. Thus for large $\left|\Gamma_{U}\right|$, the error is due primarily to the second term.

For completeness, it is desirable to take a more careful look at the approximate relationship, (10). By use of techniques similar to those employed with (1), one can show that

$$
R_{S}=\left|a \Gamma_{S}\right|\left[1+\left|\frac{R_{C S}}{R_{S}}\right|^{2}-\frac{R_{C L} R_{C S}^{*}+R_{C L}^{*} R_{C S}}{2 R_{S}^{2}}+\cdots\right] .
$$

Thus the error in (10) is of second order provided that $\left|\Gamma_{S}\right|=1$. In general, one has two choices for assigning a value to $|a|$. First, as suggested above, one can use $R_{S}$, in which case $|a|$ will be in error by the factor (1 $\left.\left|\Gamma_{S}\right|\right)$. The other alternative is to use (16) in conjunction with a fixed short, for which the deviation from unity should be negligible. In this case, $|a|$ may be in error by as much as $\pm|a|\left|R_{C S} / R_{S}\right|$. Ordinarily, the choice should be made in favor of the smaller error.

\section{Computer-Aided Solution}

As described above, the "solution" includes certain techniques which are often used in the context of problems of this type. These include the use of approximations and a change of variables (12). The objective, of course, is to reduce the amount of algebra required (which ultimately limits the applicability of these methods). In the aftermath of this effort, an alternative, more complete solution was obtained via computer methods, which will now be briefly described. From (6) and (7) one has

$$
\frac{R_{C S}}{R_{S}}=\frac{a\left(b-c^{*}\left|\Gamma_{S}\right|^{2}\right)}{|a||1-b c|\left|\Gamma_{S}\right|}
$$

and

$$
\frac{R_{C L}}{R_{S}}=\frac{a\left(b-c^{*}\left|\Gamma_{L}\right|^{2}\right)\left(1-|c|^{2}\left|\Gamma_{S}\right|^{2}\right)}{|a||1-b c|\left|\Gamma_{S}\right|\left(1-|c|^{2}\left|\Gamma_{L}\right|^{2}\right)}
$$

from which

$$
b-c^{*}\left|\Gamma_{S}\right|^{2}=|1-b c|\left|\Gamma_{S}\right| R_{C S} / R_{S}
$$

and

$$
b-c^{*}\left|\Gamma_{L}\right|^{2}=\frac{|1-b c|\left|\Gamma_{S}\right|\left(1-|c|^{2}\left|\Gamma_{L}\right|^{2}\right) R_{C L}}{\left(1-|c|^{2}\left|\Gamma_{S}\right|^{2}\right) R_{S}}
$$

where the arguments of $R_{C S}$ and $R_{C L}$ have been redefined to "absorb" the phase factor $a /|a|$. (In the final result only the magnitudes of $R_{C S}$ and $R_{C L}$ are of interest.)

Conceptually, it is only necessary at this point to solve (21) and (22) for $b$ and $c$ and substitute in (3) to obtain the desired result. Apart from the factor $|1-b c|$ and those which involve $|c|^{2}$ this would be a simple exercise. Their presence, however, renders a closed-form solution difficult, if not impossible. It is a fairly simple task to define an iterative solution, however. Returning to (21) and (22), one begins by setting $|1-b c|,\left(1-|c|^{2}\right.$ $\left.\left|\Gamma_{S}\right|^{2}\right)$, and $\left(1-|c|^{2}\left|\Gamma_{L}\right|^{2}\right)$ equal to unity and then solving for $b$ and $c$. These values are then used to obtain an improved "estimate" of $|1-b c|$, etc., and the procedure is repeated. Because the magnitudes of $b$ and $c$ are small, the procedure quickly converges, although a substantial amount of algebra is required.

It is possible, however, to effect the algebra by means of certain routines which were developed as an accessory to a previously described computer program [1], [2] for the analysis of microwave systems. Although the details are the subject of a paper to follow, the basic function of these routines is to perform the basic operations of addition, subtraction, multiplication, division, absolute value, etc., on the output of this program, which is in the form of a Taylor series power expansion.

By use of these methods, it was possible to include the effects of a nonzero reflection for the sliding termination, and a nonunity reflection from the moving short. Based on this result, a more complete expression may be written:

$$
\begin{gathered}
\frac{P_{3}}{P_{4}}=\left|a \Gamma_{U}\right|^{2}\left[1 \pm 2\left|\frac{R_{C L}}{R_{S}}\right| \frac{\left|\Gamma_{S}\right|^{2}-\left|\Gamma_{U}\right|^{2}}{\left|\Gamma_{S} \Gamma_{U}\right|}\right. \\
\left. \pm 2\left|\frac{R_{C S}}{R_{S}}\right|\left|\frac{\Gamma_{U}}{\Gamma_{S}}\right|+\left|\frac{R_{C L}}{R_{S}}\right|^{2} \mid \frac{\left|\Gamma_{S}\right|^{2}}{\left|\Gamma_{U}\right|^{2}}\right]
\end{gathered}
$$

which may be compared with (16). Not included in this expression are an additional 17 second-order terms and the 50 third-order terms, which also include the contribution from the nonzero sliding termination. 


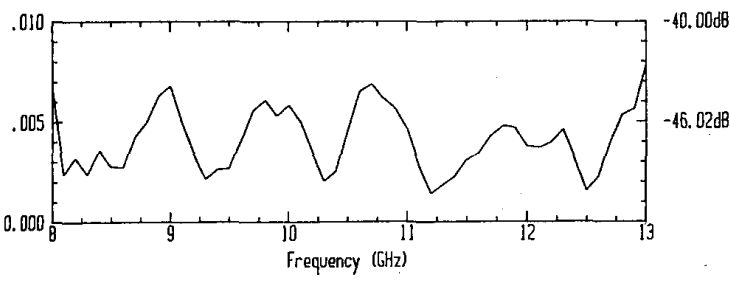

Fig. 3. Magnitude of $b$ (directivity term).

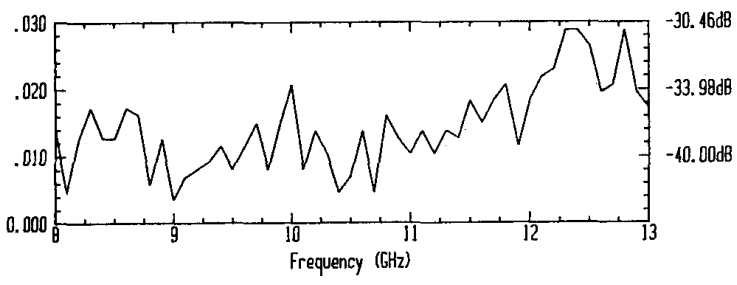

Fig. 4. Magnitude of $d$.

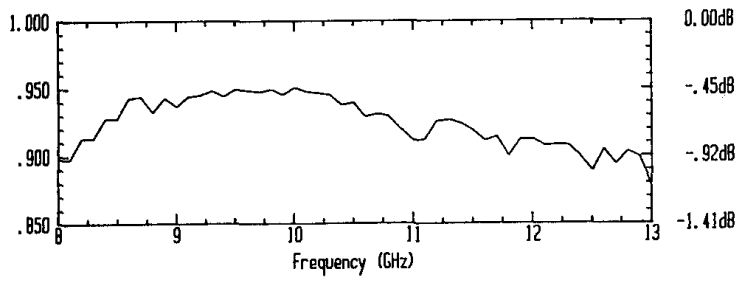

Fig. 5. Magnitude of $a$ (scaling factor).

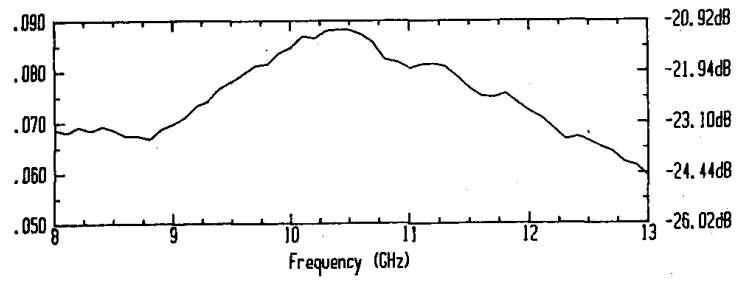

Fig. 6. Reflection factor for degraded sliding load.

\section{ExPERIMENTAL Results}

Measurements were made with an HP 8755P automatic scalar network analyzer using an $X$-band waveguide reflectometer with two $10-\mathrm{dB}$ directional couplers.

With sliding terminations, the maximum and minimum values of $|w|$ are obtained from (5) to (7):

$$
|w|_{\max }=R+\left|R_{C}\right|
$$

and

$$
|w|_{\min }=|R-| R_{C}||
$$

From (10) and (11), $R_{S}>\left|R_{C S}\right|$ for the sliding short. Then

$$
\left(|w|_{\max }+|w|_{\min }\right) / 2=R_{S}=|a|
$$

and

$$
\frac{\left(|w|_{\max }-|w|_{\min }\right)}{\left(|w|_{\max }+|w|_{\min }\right)}=\frac{\left|R_{C S}\right|}{R_{S}} \simeq|d| .
$$

For the sliding load, $R_{C L}$ and $R_{L}$ are given approximately by (8) and (9). With the equipment used in these measurements, $\left|R_{C L}\right|$ and $R_{L}$ were comparable in magnitude, and less than 0.1 . In order to identify which was which from the measurements (24) and (25), the sliding load was degraded by attaching a small piece of copper foil to the sliding element. The size and the position of the copper tape were determined by trial and error, with the goal being a nearly constant reflection factor less than, but close to, 0.1 in the entire frequency range.

For the degraded sliding load $R_{L}>\left|R_{C L}\right|$. Then,

$$
\left(|w|_{\max }+|w|_{\min }\right) / 2=R_{L} \simeq\left|a \Gamma_{L}\right|
$$

and

$$
\left(|w|_{\max }-|w|_{\min }\right) / 2=\left|R_{C L}\right| \simeq|a b| .
$$

Since $|a|$ is known from the measurement of the sliding short, these equations determine $|b|$ and $\left|\Gamma_{L}\right|$.

The measurements were made under computer control. The sliding terminations were set at 20 different positions. For each position the frequency was stepped by $0.1 \mathrm{GHz}$ from 8 to $13 \mathrm{GHz}$. From these data the computer determined the maximum and minimum values and calculated and plotted the results shown in Figs. 3-6. It will be noted that $|b|$ and $|d|$ (or $\left|R_{C L}\right| / R_{S}$ and $\left|R_{C S}\right| / R_{S}$ ) remain below 0.01 and 0.03 , respectively, while the typical values are perhaps half of these figures or less. The substitution of these results in (16) or (23) gives errors which range from 10 percent down to 3 percent for $0.1 \leq\left|\Gamma_{U}\right| \leq 1$, and with a rapid increase in the error for smaller values of $\left|\Gamma_{U}\right|$.

Separate manual measurements were made in order to confirm that the maximum and minimum values, as determined by the described procedure, were indeed a good approximation. The manual measurements and repeated computer-controlled measurement gave results that were everywhere within \pm 0.0025 of the results in Fig. 3 and within \pm 0.003 of the results in Fig. 4 .

\section{REFERENCES}

[1] B. Guldbrandsen, "A computer program for algebraic error analysis of complex microwave systems,"' IEEE Trans. Instrum. Meas., vol IM-32, pp. 173-176, Mar. 1983.

[2] - "Computer-aided algebraic analysis of systematic errors in a composite microwave system," Proc. IEEE, vol. 74, pp. 68-71, Jan. 1986. 